\title{
Describing resonances in a discrete basis
}

\author{
M. Rodríguez-Gallardo, J. M. Arias, and J. Gómez-Camacho \\ Departamento de Física Atómica, Molecular y Nuclear, Facultad de Física, Universidad de Sevilla, Apartado 1065, \\ 41080 Sevilla, Spain
}

(Received 10 October 2003; published 4 March 2004)

\begin{abstract}
The problem of describing resonances when the continuum is represented by a discrete set of normalizable states is addressed. In particular, here the description of resonances in a transformed harmonic oscillator basis is presented. A method to disentangle the resonances from the nonresonant continuum is proposed. The Ginocchio potential is used to model a case in which resonances appear in the continuum and a reference case in which only nonresonant continuum appears.
\end{abstract}

DOI: 10.1103/PhysRevC.69.034308

PACS number(s): 24.30.-v, 03.65.Ca, 24.10.Eq

\section{INTRODUCTION}

Recently there has been much interest in investigating the effect of the continuum in the properties of quantum systems. In nuclear physics, the advent of unstable beam facilities has allowed the study of systems which have a very small binding energy. The properties of such weakly bound nuclei are strongly affected by the states in the continuum and, consequently, both bound and unbound states have to be included in any realistic description of the system. In molecular physics, new experimental laser techniques have allowed to reach energy regions where chemical activity is significant. This implies breaking of molecular bonds in order to produce new rearrangement or even dissociation. In such a situation the continuum part of the spectrum plays an important role and its effects cannot be neglected.

An explicit consideration of the continuum in structure and reaction calculations is made difficult because the continuum wave functions have an infinite range and are not normalizable. Because of that it is necessary to use some discretization procedure to substitute the continuum of breakup states by a finite number of normalizable states which, in the adequate limit, should represent the effect of coupling to the true continuum. There are in the literature several discretization procedures that have been used in nuclear, molecular, and atomic physics each one with advantages and drawbacks. Here we will mention few of them. The Gamow states [1] are non-normalizable solutions of the Schrödinger equation corresponding to outgoing boundary conditions characterized by complex energies. The method of continuum discretization coupled channels [2] discretizes the continuum by means of taking fixed intervals, or bins, of $k$ values in the continuum states. A Sturmian basis is obtained when one uses bound states of scaled potentials which are orthogonal when weighted with the potentials [3-5]. The Gaussian expansion method takes a nonorthogonal basis composed of Gaussian functions in geometric progression [6]. A complete basis of single particle wave functions, such as the harmonic oscillator, can be used to expand both bound and scattering states [7].

We have recently proposed an alternative procedure, based on the use of a transformed harmonic oscillator (THO) basis, to describe the effect of the continuum [8-11]. The basic idea in the THO method is to define a local scale trans- formation [12-14] which is such that converts the bound ground state radial wave function of the weakly bound system $\phi_{B}(r)$ into the ground state harmonic oscillator radial wave function $\phi_{0}^{H O}(s)=(4 / \sqrt{\pi})^{1 / 2} s \exp \left[-s^{2} / 2\right]$ [8-11]. The function $s(r)$ defines the local scale transformation and it is given for a three-dimensional problem under central forces by

$$
\int_{0}^{r}\left|\phi_{B}\left(r^{\prime}\right)\right|^{2} d r^{\prime}=\frac{4}{\sqrt{\pi}} \int_{0}^{s} s^{\prime 2} \exp \left[-s^{\prime 2}\right] d s^{\prime} .
$$

Then, by using the generalized Laguerre polynomials $L_{n}^{\alpha}(z)$ one generates a set of orthogonal wave functions $\psi_{n}^{T H O}(r)=L_{n}^{1 / 2}\left([s(r)]^{2}\right) \phi_{B}(r)$, such that the state with $n=0$ coincides with the bound ground state, and the states with $n>0$ describe the continuum, or other bound states if they exist. Then, one takes a finite basis, which is uniquely determined by the number $N$ of THO states considered, and diagonalizes the Hamiltonian in this basis. The resulting eigenstates and eigenvalues are taken as representatives of the continuum. We showed in previous papers [8-11] that, as the number of states in the THO basis increases, the eigenstates appear densely packed close to the breakup threshold, although there are eigenstates that appear at higher energies. Besides, we demonstrated that global structure magnitudes related to the coupling to the continuum, such as sum rules, were very accurately described using relatively small THO basis.

The THO method does not explicitly take into account the structure of the continuum in the discretization procedure. Thus, the only information which is required in order to build the THO basis, and even to evaluate the matrix elements of the Hamiltonian, is the ground state wave function. However, it is known that not all the continuum states couple equally to the ground state. In general, the continuum states which are near to the resonances play a much more important role than the nonresonant continuum. This is related to the fact that, when the energy is close to a resonance, the probability that the continuum state is in the range of the potential is larger than for the nonresonant part.

In this paper we address the question of how adequate is the THO basis to describe the resonant structure of the continuum. For that purpose, we use the Ginocchio potential [15], which can be solved analytically and has resonances 
even for $L=0$ states. The Pöschl-Teller (PT) potential [16], which does not have resonances, is a particular case of the Ginocchio potential. We will compare the continuum states of the PT potential with those of a Ginocchio $(\mathrm{G})$ potential that presents resonant states, to investigate the effect of the resonances in the continuum discretization. In Sec. II we review the Ginocchio potential, and present the results of continuum discretization in the G and PT cases. In Sec. III we present a procedure to obtain the resonance wave function from the THO basis. Section IV is for discussion on the coupling of the ground state to the continuum. Finally, Sec. $\mathrm{V}$ is for summary and conclusions.

\section{CONTINUUM DISCRETIZATION FOR RESONANT AND NONRESONANT POTENTIALS}

We consider the $L=0$ bound and continuum states of a two-body system characterized by a reduced mass $m$ and an interaction $U(R)$. The radial wave functions $\phi(R)$ are eigenstates of the Hamiltonian

$$
H=-\frac{\hbar^{2}}{2 m} \frac{d^{2}}{d R^{2}}+U(R) .
$$

The physical variable $R$ can be taken as $R=S r$, where $r$ is a dimensionless quantity and $S$ sets the length scale of the problem. Similarly, the potential $U(R)$ can be written as $\left(\hbar^{2} / 2 m S^{2}\right) v(r)$, where $v(r)$ is a dimensionless potential and $\hbar^{2} / 2 m S^{2}$ sets the energy scale of the problem. So, a dimensionless Hamiltonian can be written as

$$
h=-\frac{d^{2}}{d r^{2}}+v(r) \text {. }
$$

The Ginocchio potential has been described in the literature [15]. In this paper we will make use of a simplified version of it, which may be written as

$$
\begin{aligned}
v(r)= & -\lambda^{2} \nu(\nu+1)\left(1-y^{2}\right)+\left(\frac{1-\lambda^{2}}{4}\right)\left(1-y^{2}\right)\left[2-\left(7-\lambda^{2}\right) y^{2}\right. \\
& \left.+5\left(1-\lambda^{2}\right) y^{4}\right],
\end{aligned}
$$

where

$$
r(y)=\frac{1}{\lambda^{2}}\left\{\operatorname{arctanh}(y)+\left[\lambda^{2}-1\right]^{1 / 2} \arctan \left(\left[\lambda^{2}-1\right]^{1 / 2} y\right)\right\} .
$$

Thus, the potential depends on two parameters $\lambda$ and $\nu$. When $\lambda=1$, the potential becomes the Pöschl-Teller potential

$$
v(r)=-\frac{\nu(\nu+1)}{\cosh ^{2}(r)} .
$$

When the parameter $\lambda$ has sufficiently large values, it produces a potential barrier, and generates resonant states in the continuum. The parameter $\nu$ is related to the number of bound states that the potential supports. When $1<\nu<3$, the system supports just one bound state with $L=0$.

We take as a nonresonant reference case the Pöschl-Teller potential with $\nu=2$. This has only one bound state, whose

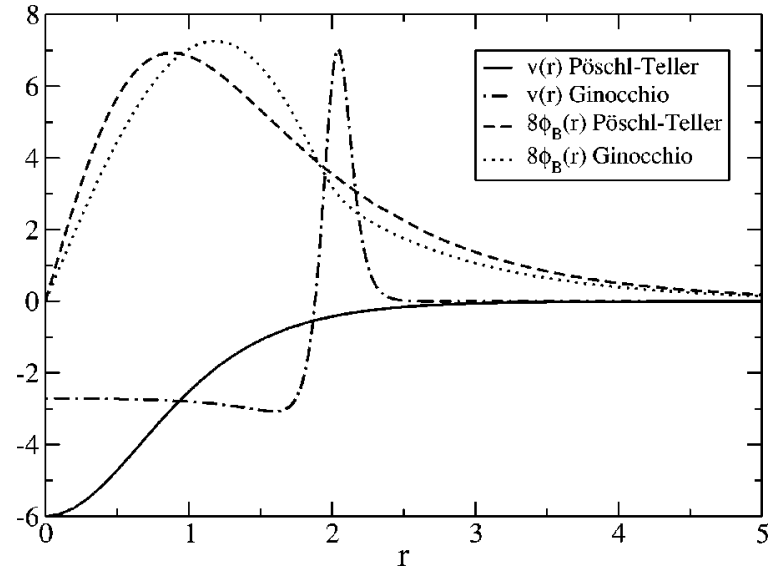

FIG. 1. Ginocchio $(\lambda=10$ and $\nu=1.58)$ and Pöschl-Teller $(\lambda=1$ and $\nu=2$ ) potentials, together with the corresponding ground state wave functions. The wave functions have been multiplied by 8 in order to display them and the potentials in the same figure. (All quantities plotted are dimensionless.)

energy is (in dimensionless units) $e_{B}=-1$ and its mean square radius is 1.35 . As a resonant case, we take a Ginocchio potential whose parameters are $\lambda=10$ and $\nu=1.58$. This potential has also one bound state. Choosing the appropriate length scale this state has energy $e_{B}=-1$ and mean square radius 1.35 . These potentials, along with the corresponding wave functions for the bound ground state, are plotted in Fig. 1. These two wave functions (labeled PT and G hereafter), as mentioned above, have the same binding energy and mean square radius. The difference between them is related to the fact that, while the PT potential has no resonances, the G potential has resonances, the first three of which have energies $4.55,14.99$, and 30.08 and widths $2.32,6.59$, and 12.73 . The difference between the two bound wave functions is related to the larger slope with which the $\mathrm{G}$ wave function decreases in the range of values which corresponds to the barrier $(r \approx 2)$.

We have calculated the local scale transformations $s(r)$ which convert the PT and G wave functions in the ground state $\mathrm{HO}$ wave function. These transformations are given by Eq. (1). The results are plotted in Fig. 2. It should be noticed that the difference between the resonant and the nonresonant cases is reflected in the curvature of the function $s(r)$ for the resonant case at the distance corresponding to the barrier.

Once the local scale transformation is obtained, the THO basis can be built for both cases as

$$
\langle r \mid T H O, n\rangle=\psi_{n}^{T H O}(r)=C_{n} L_{n}^{1 / 2}\left([s(r)]^{2}\right) \phi_{B}(r),
$$

where the normalization constant is

$$
C_{n}=\sqrt{\frac{\pi^{1 / 2} \Gamma(n+1)}{2 \Gamma(n+3 / 2)}} .
$$

The Pöschl-Teller and Ginocchio potentials have analytic continuum wave functions [15]. Thus, the overlap of the THO wave functions with the true continuum states $\phi(k, r)$ can be expressed as 


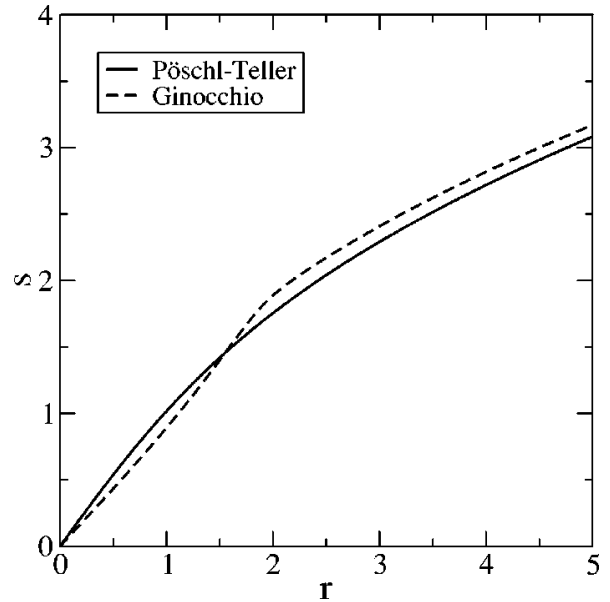

FIG. 2. Local scale transformations $s(r)$ for the PT and G ground state wave functions. (All quantities are dimensionless.)

$$
\langle k \mid T H O, n\rangle=\psi_{n}^{T H O}(k)=\int d r \psi_{n}^{T H O}(r) \phi(k, r) .
$$

Note that in our units the energy is just $k^{2}$. For large distances, beyond the range of the potential, the continuum wave functions behave as

$$
\phi(k, r) \rightarrow \sqrt{\frac{2}{\pi}} \sin \left(k r+\delta_{k}\right),
$$

and they fulfil the orthogonality condition

$$
\int d r \phi(k, r) \phi\left(k^{\prime}, r\right)=\delta\left(k-k^{\prime}\right) .
$$

It is convenient to define an operator $P(N)$ which projects into the space generated by the THO states from $n=0$ to $N$. Note that the state with $n=0$ is the bound ground state of the Hamiltonian. So,

$$
P(N)=\sum_{n=0}^{N}|T H O, n\rangle\langle n, T H O| .
$$

Note that as $N$ tends to infinity the THO basis tends to be complete and $P(N)$ approaches the unity operator.

Remember that we are considering cases in which there is just one bound state which is decoupled from the rest by the Hamiltonian. Thus, considering the THO basis $\{|T H O, n\rangle, n$ $=0, \ldots, N\}$ implies having $N$ states describing the continuum. The Hamiltonian can be diagonalized in such a basis obtaining its eigenstates and eigenvalues. This is formally equivalent to diagonalize $P(N) h P(N)$. The eigenstates in the continuum will be orthonormal combinations of the states in the THO basis given by

$$
|T H O, N, h ; i\rangle=\sum_{n=1}^{N}\langle T H O, n \mid N, h ; i\rangle|T H O, n\rangle, \quad i=1, \ldots, N,
$$

which satisfy

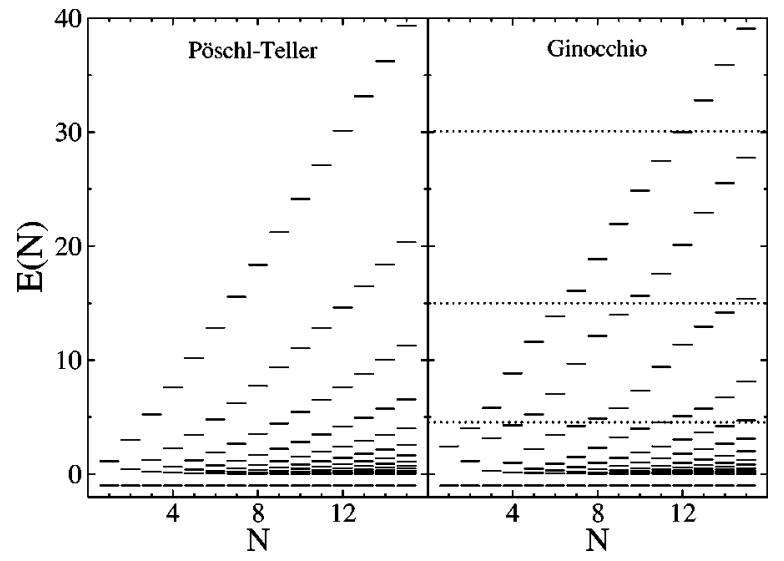

FIG. 3. Energy levels in the THO basis for the PT and G Hamiltonians as a function of the number of states included in the THO basis. The known energies of the first three resonances in the $\mathrm{G}$ case are shown by dotted lines. (All quantities in the plot are dimensionless.)

$$
\begin{aligned}
& \sum_{n=1}^{N}\langle\text { THO }, m|h| T H O, n\rangle\langle T H O, n \mid N, h ; i\rangle \\
& \quad=E(N ; i)\langle T H O, m \mid N, h ; i\rangle .
\end{aligned}
$$

In Fig. 3 we plot the energy levels $E(N ; i)$ obtained from the diagonalization of both Hamiltonians, PT and G, as the dimension of the THO basis is increased. In both cases, the structure of the energy levels is very similar. The density of states is larger close to the breakup threshold, which occurs at $E=0$. However, a closer inspection of the two figures indicates that while the level density decreases continuously in the nonresonant PT case as the energy increases, the levels have a slight tendency to cluster around the energies of the resonances in the G case. It was shown in Ref. [17] that, for the case of narrow resonances, the diagonalization of the Hamiltonian in a discrete basis with sufficient (although not too large) dimension gives some eigenstates whose energies are very stable as the dimension of the basis is increased, and this can be taken as a signature for the resonances. However, this is not the case for the relatively wide resonances considered here.

The exploration of Fig. 3, and the inspection of the eigenstates of the Hamiltonian in the THO basis indicate that the resonant states do not appear explicitly as eigenstates of the Hamiltonian. This is shown in Fig. 4, where the radial wave functions of the two Hamiltonian eigenstates with energies close to the energy of the first resonance in the $G$ potential are plotted. It can be seen that none of them present a clear resonant character. For a resonance one expects a large presence probability at small distances reflected in a large amplitude of the wave function inside the potential well. In Fig. 4, it is clear by visual inspection that this is not the case. Thus, one should conclude that, in a discrete basis, the resonant character is distributed among several states with energies in the vicinity of the resonance.

This can be seen even more clearly by showing the overlap of the eigenstates of the Hamiltonian in the discrete basis 


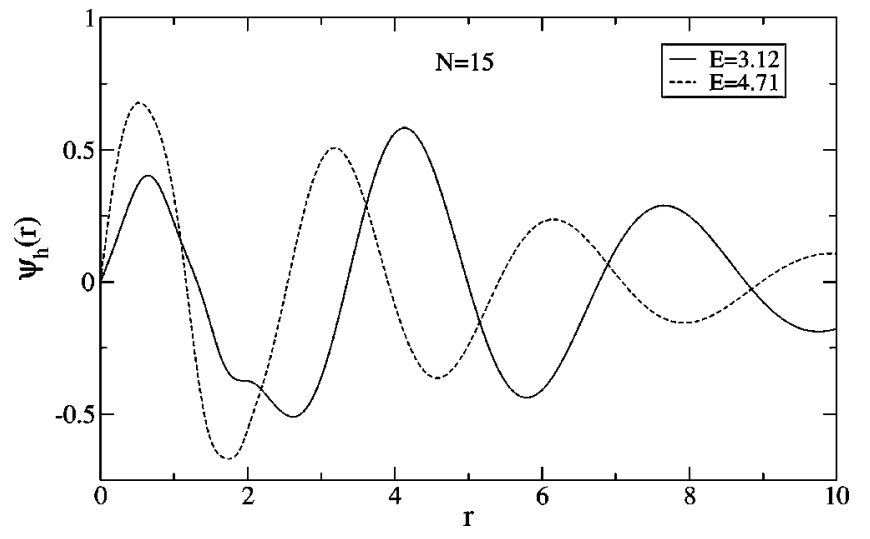

FIG. 4. Wave functions for the Hamiltonian eigenstates in a THO basis with $N=15$ with the energy closer to the first resonance, $E=4.55$. (All quantities plotted are dimensionless.)

with the true continuum states. For a resonance, one expects to have a Lorentzian distribution in energy, which corresponds to an asymptotic momentum distribution given by

$$
\rho(k) d k=\frac{k}{\pi} \frac{\Gamma}{\left(k^{2}-E_{r}\right)^{2}+(\Gamma / 2)^{2}} d k
$$

In Fig. 5, this Lorentzian form is compared with the momentum distribution of the two eigenstates of the Hamiltonian that have eigenvalues close to the energy of the first resonance. There it can be seen that the momentum distributions of these two eigenstates of the Hamiltonian do not resemble the expected form for a resonance, although both are close to it in energy.

However, we expect that the THO basis should be able to describe the resonances. This is because the THO basis can describe accurately the Hilbert space that corresponds to the range of distances within the potential, which is the most relevant for the resonances. To see this more explicitly, we have plotted in Fig. 6 the sum of the momentum distributions of all the states in the THO basis,

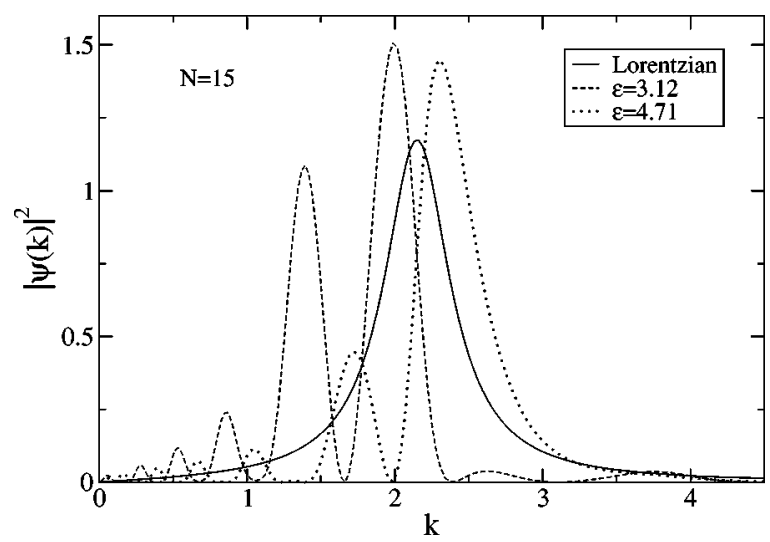

FIG. 5. Momentum distributions for the Hamiltonian eigenstates in a THO basis with $N=15$ with the energy close to the first resonance in the $\mathrm{G}$ potential, compared to the momentum distribution expected for a resonance (Lorentzian shape). (All quantities are dimensionless.)

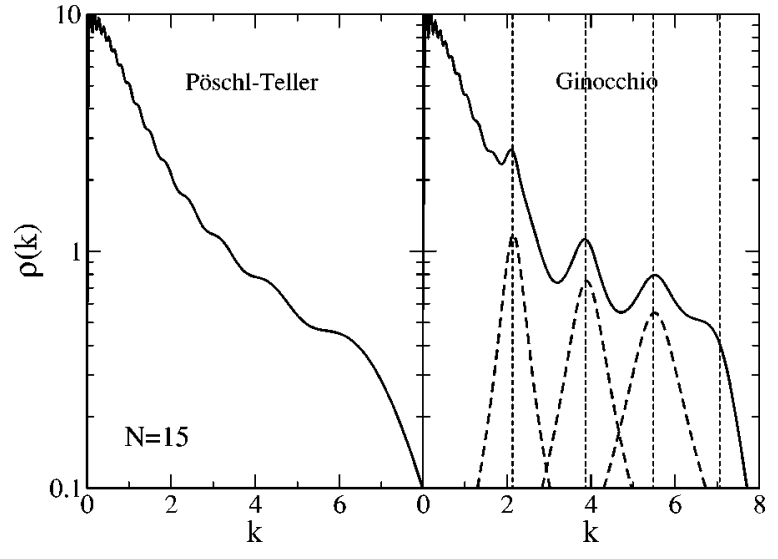

FIG. 6. Momentum distribution $\rho(k)$ for the PT and G potentials for $N=15$. The momentum distributions for the first three resonances of the $\mathrm{G}$ potential are also shown in bold dashed lines. The thin dashed lines indicate the position of the resonances. (All quantities are dimensionless.)

$$
\rho(k)=\langle k|P(N)| k\rangle=\sum_{n=1}^{N} \psi_{n}^{T H O}(k)^{2} .
$$

This sum is a measurement of the completeness of the THO basis in momentum space. If the basis was truly complete, $\rho(k)$ would be infinite for all the values of $k$. So, for each value of $k$, the value of $\rho(k)$ tells us how well suited is the basis to describe that momentum range. It can be seen in Fig. 6 that for the nonresonant case the function $\rho(k)$ reduces monotonically as $k$ increases, for the resonant case it has relative maxima which corresponds exactly to the resonances, both in position and width.

So, we can conclude that the resonant states should be well described within the Hilbert space generated by the THO basis, although they do not correspond to eigenstates of $h$ in that basis. In the following section, we describe a procedure to disentangle the resonances from the nonresonant continuum in the THO basis.

\section{DISENTANGLING THE RESONANCES IN THE THO BASIS}

When there are resonances, the continuum states have different behavior depending on whether their energy is close to one of them or not. The continuum states whose energy is close to a resonance have a relatively large probability density of being in the interior of the potential well. Moreover, all the continuum states whose energy is close to a resonance have a similar shape in the interior of the potential, although they oscillate differently outside of the potential.

The THO basis should be, when the size of the basis is sufficiently large, an adequate representation of the continuum. Our aim in this section is to find a single discrete state in the THO basis which represents the behavior of the continuum states within the potential well at energies around a resonance. This state will not be, in general, an eigenstate of the Hamiltonian in the THO basis as shown in the preceding section. 
To build the resonances in the THO basis we consider the eigenstates of the operator $\Delta^{2}$ defined by the expression

$$
\begin{aligned}
\Delta^{2} & =P(N)\left(h-e_{B}\right) Q(N)\left(h-e_{B}\right) P(N) \\
& =P(N)\left(h-e_{B}\right)^{2} P(N)-\left[P(N)\left(h-e_{B}\right) P(N)\right]^{2},
\end{aligned}
$$

where $h$ is the Hamiltonian of the system, $e_{B}$ is the ground state energy, and $Q(N)=1-P(N)$ is the operator that projects out of the THO basis. The meaning of the operator $\Delta^{2}$ is the following: The expectation value of $\Delta^{2}$ in a state belonging to the subspace generated by the THO basis measures how strongly does the Hamiltonian couple this state to the states outside the THO subspace. If the operator $\Delta^{2}$ (which is positive definite) is diagonalized in the THO basis, then the eigenstates which have small eigenvalues will correspond to states which are weakly coupled to the states outside the THO basis. So, the operator $\Delta^{2}$ has the interpretation of the square of an energy width, associated with the fact that the THO states will eventually evolve to abandon the THO subspace. Thus, $\hbar / \sqrt{\Delta^{2}}$ would be related to the time a given state remains in the space generated by the THO basis, which is also related to the time that a given state remains in the vicinity of the potential.

We expect that a resonance, as opposed to the nonresonant continuum with similar energy, should remain for a longer time at the vicinity of the potential. These configurations are well described in the THO basis. Thus, the state that describes the resonance in the THO basis should not couple strongly to states outside the THO basis. Hence, we should look for the resonance between the eigenstates of $\Delta^{2}$ which have low eigenvalues.

On the other hand, as Fig. 6 shows, the THO basis describes more accurately the states with low excitation energy, resonant or not. Hence, not all the eigenstates of $\Delta^{2}$ with low eigenvalues correspond to resonances. The relevant states are those with low values of $\Delta^{2}$, but which have, at the same time, relatively high values of the energy.

The eigenstates of $\Delta^{2}$ will be orthonormal combinations of the states in the THO basis given by

$$
\begin{aligned}
\left|T H O, N, \Delta^{2} ; i\right\rangle= & \sum_{n=1}^{N}\left\langle T H O, n \mid N, \Delta^{2} ; i\right\rangle|T H O, n\rangle, \\
& i=1, \ldots, N,
\end{aligned}
$$

which satisfy

$$
\begin{aligned}
& \sum_{n=1}^{N}\left\langle T H O, m\left|\Delta^{2}\right| T H O, n\right\rangle\langle T H O, n \mid N, h ; i\rangle \\
& \quad=\Delta^{2}(N ; i)\left\langle T H O, m \mid N, \Delta^{2} ; i\right\rangle .
\end{aligned}
$$

In Fig. 7 we have plotted, for the G and PT cases, the expectation value of $h$ for the eigenstates of $\Delta^{2}$, as a function of the dimension of the basis. We have joined by lines the values corresponding to the lowest eigenstates of $\Delta^{2}$ for each value of $N$, then the next one, and so on. Note that as $N$ increases along a given line, the eigenvalue of $\Delta^{2}$ decreases, and, consequently, the time that the state remains in the vicinity of the potential increases. For the nonresonant poten-

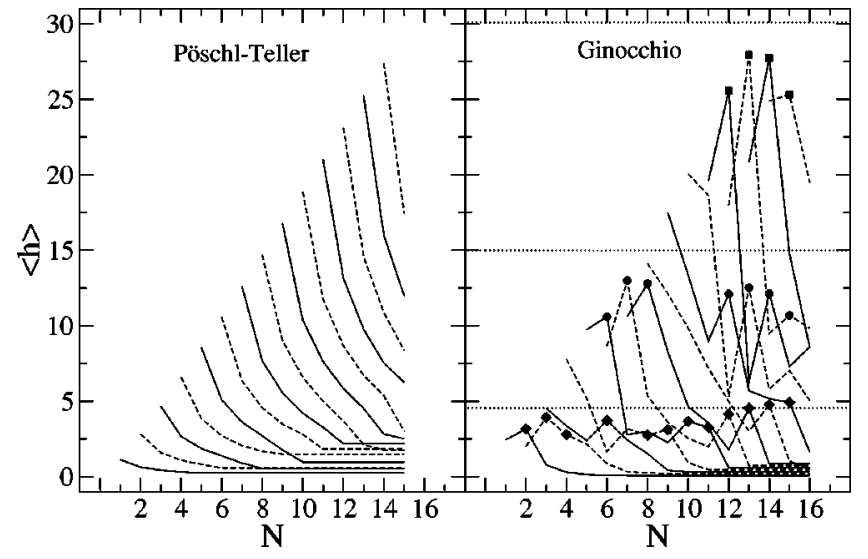

FIG. 7. Expectation value of the energy for the eigenstates of $\Delta^{2}$ in a THO basis as a function of the dimension of the basis for the PT and G cases. The resonances are marked by special symbols. The known energies of the first three resonances in the $\mathrm{G}$ case are shown by dotted lines. (All quantities are dimensionless.)

tial, we can see that the expectation values of $h$ decrease monotonically as $N$ increases. This is expected because a longer time should be associated with a smaller velocity and hence a smaller energy. However, for the resonant potential, it can be seen that the trend is not always the same. There are some cases in which an increase in $N$, and hence a decrease in the value of $\Delta^{2}$, or an increase in the time of permanence close to the potential, is accompanied by an increase in the expectation value of the energy. In Fig. 7 we have marked with symbols the states we have identified as resonances because they have a large probability of being within the potential. As it can be seen in Fig. 7, there is a clear correspondence between resonances and eigenstates of $\Delta^{2}$ which have an anomalously large expectation value of the energy. Also, it should be noticed that the expectation value for the energy is consistent with the known average energy of the resonance. So, we conclude that resonances in the THO basis can be obtained as the eigenstates of $\Delta^{2}$ with a large energy, which deviates from the decreasing tendency as $N$ is increased. The wave functions of the resonance are given by

$$
\psi_{\text {res }}(r)=\sum_{n=1}^{N}\left\langle T H O, n \mid N, \Delta^{2} ; i\right\rangle \psi_{n}^{T H O}(r)
$$

in configuration space, and

$$
\psi_{r e s}(k)=\sum_{n=1}^{N}\left\langle T H O, n \mid N, \Delta^{2} ; i\right\rangle \psi_{n}^{T H O}(k)
$$

in momentum space. In this paper, since we are keeping a small dimension of the THO basis, we expect to get a good description only of the first resonance. However, we also show in Fig. 7 the second and third resonances in the G potential, which appear at energies consistent with the known values.

We have investigated the robustness of this method as the size of the basis is increased. In Fig. 8 we plot the identified first resonant wave function (upper panel, $i=1$ ) as the size of 


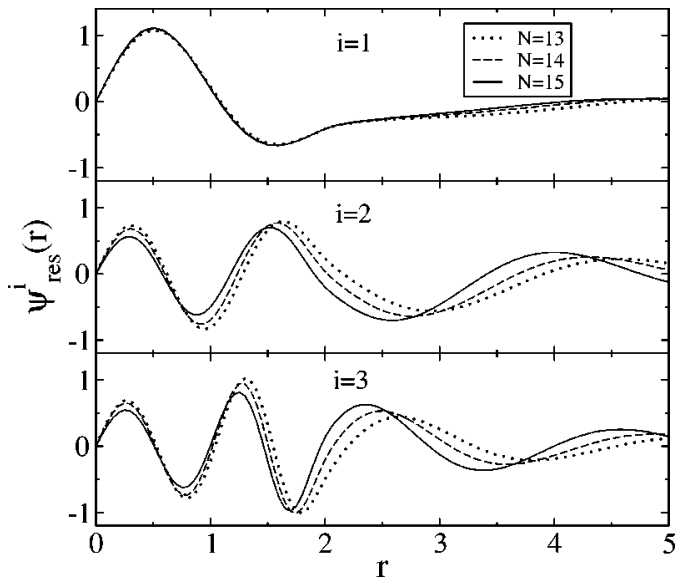

FIG. 8. THO resonances for different values of the basis size: $N=13,14$, and 15 . The upper panel is for the first, the middle panel for the second, and the lower panel for the third resonances. (All quantities are dimensionless.)

the basis is increased $(N=13,14,15)$ we see that the form of the wave function in the interior of the potential well is practically unaffected. We show in this figure, the second (middle panel, $i=2$ ) and the third resonances (lower panel, $i=3$ ) too. It can be seen that also these resonances are relatively stable inside the well although they are changing more than the first one due to the size of the basis that covers well the energy region of the first resonance but scarcely the energy region of the other resonances.

We have performed several checks to see that the resonances we obtain from the eigenstates of $\Delta^{2}$ in the THO basis correspond to other definitions of resonances. First, we have compared our resonance with the actual continuum wave function at the energy $E=E_{r}$. We see in Fig. 9 that the two wave functions coincide within the range of the potential well. Outside of it, the THO wave function vanishes, while the continuum state oscillates. It is remarkable that the two wave functions show similar values within the potential, although they have different normalization criteria. While the resonant wave function in the THO basis is normalized so that the integral of the square of the wave function is one, the

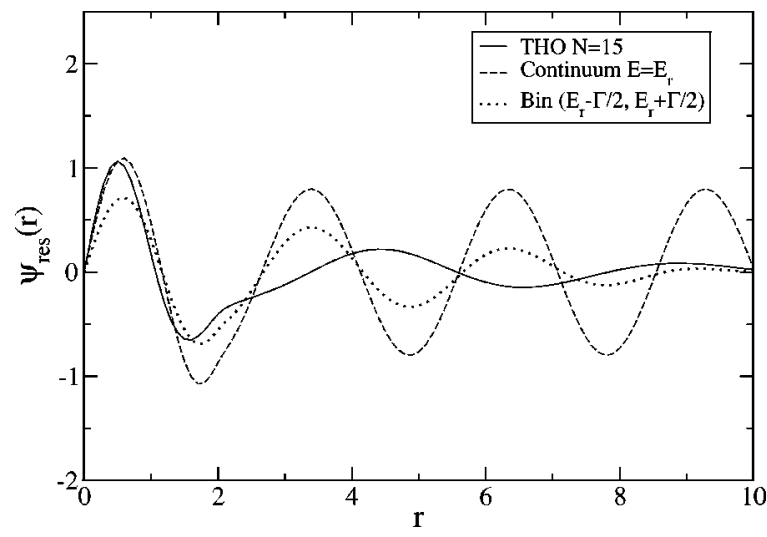

FIG. 9. THO resonance for a basis $N=15$ compared with the actual continuum wave function, and the bin wave function. See the text for details. (All quantities are dimensionless.)

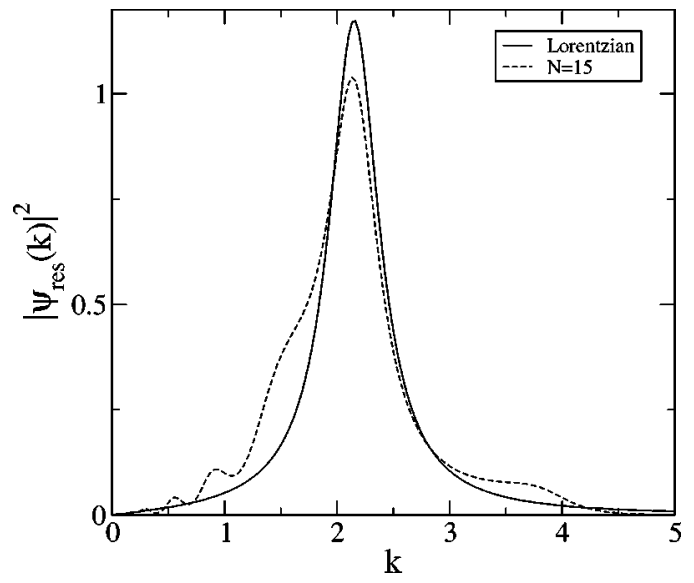

FIG. 10. Momentum distribution for the first THO resonance with $N=15$, compared to the expected resonance distribution (Lorentzian shape). (All quantities plotted are dimensionless.)

continuum wave functions satisfy Eq. (11). However, any wave packet which is constructed with the continuum wave functions at energies close to the resonance is square normalizable, and hence it can be compared to the THO resonance. Thus, we have also compared in Fig. 9 the THO resonance with an average of continuum wave functions (a bin) which is taken in the range from $E=E_{r}+\Gamma / 2$ to $E=E_{r}-\Gamma / 2$ ( $\Gamma$ is the width of the resonance).

In Fig. 10, we compare the Lorentzian form expected for the first resonance with the momentum distribution of this resonance in the THO basis with $N=15$. There it can be seen that the momentum distribution does resemble the expected form for a resonance. Note also that both the position and width of the resonance is well reproduced.

\section{COUPLING OF THE BOUND STATE WITH THE CONTINUUM}

In this section we consider the coupling of the ground state to the continuum. We consider the matrix elements of the operator $r$ between the bound state and the continuum states. Our aim is to investigate the difference between the resonant (Ginocchio) and nonresonant (Pöschl-Teller) cases, and to evaluate the adequateness of the THO basis to describe these differences.

We evaluate the bound-to-continuum amplitudes as

$$
A(\text { g.s. }, k)=\langle\text { g.s. }|r| k\rangle \simeq \sum_{n=1}^{N}\langle\text { g.s. }|r| T H O, n\rangle\langle T H O, n \mid k\rangle \text {. }
$$

The strength function of the operator $r$ is given by $S(k)$ $=\mid A$ (g.s.,$k)\left.\right|^{2}$. In Fig. 11, it can be clearly seen that, in the nonresonant case, the strength function is a smooth function of the momentum. However, in the resonant case a clear structure associated with the resonance appears in the range of $k$ values which correspond to the first resonance. In Fig. 11 we have plotted (full line) the exact strength function $S(k)=\mid\left. A($ g.s.,$k)\right|^{2}=\mid\left.\langle$ g.s. $|r| k\rangle\right|^{2}$ and the 


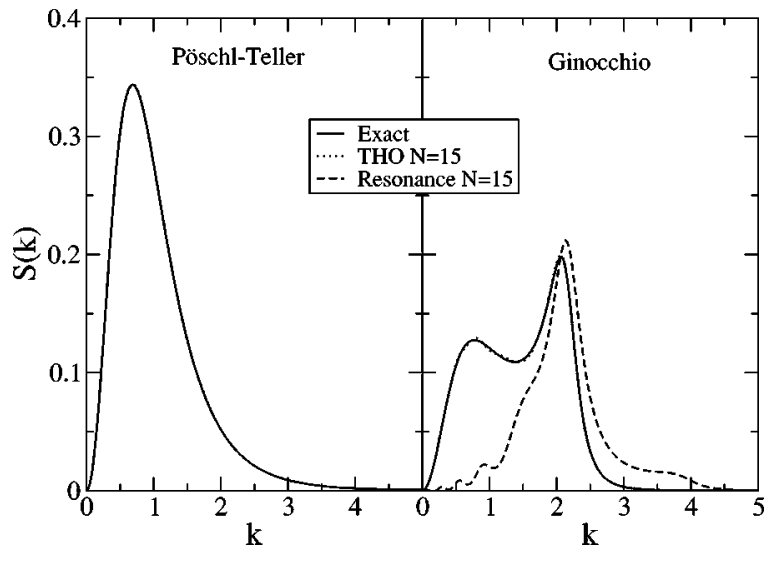

FIG. 11. Strength function $S(k)$ for the operator $r$ as a function of the momentum for the PT and G potentials. The exact values and the value obtained for a THO basis with $N=15$ (which are almost indistinguishable) are presented for both cases. The contribution of just the first THO resonance in the $\mathrm{G}$ case is also shown. (All quantities are dimensionless.)

strength function obtained for an $N=15$ THO basis $S(k)$ $=\mid A$ (g.s., $k)\left.\right|^{2}=\mid\left.\sum_{n=1}^{N}\langle$ g.s. $|r| T H O, n\rangle\langle T H O, n \mid k\rangle\right|^{2}$ (dot line). Both results are basically the same for PT and G potentials. One can evaluate the contribution to $S(k)$ of the THO resonances. The amplitude that corresponds to the resonance $i$ is given by

$$
A^{R, i}(k)=\left\langle\text { g.s. }|r| T H O, N, \Delta^{2} ; i\right\rangle\left\langle T H O, N, \Delta^{2} ; i \mid k\right\rangle .
$$

We have evaluated the contribution of the THO state which corresponds to the first resonance. This is plotted in Fig. 11 as a dashed line. The peak observed in the strength function at around $k=2$ is clearly reproduced by this single state, while the peak below $k=1$ has nothing to do with the resonance.

The contributions of the second and third resonances can also be evaluated. It should be noticed that the amplitudes of the resonances can interfere with each other, and also with the nonresonant continuum.

\section{SUMMARY AND CONCLUSIONS}

We have applied the THO method to investigate the role of resonances in the continuum. For that purpose, we have used the wave functions of the Ginocchio potential, which is analytically solvable. This potential is determined by two dimensionless parameters $\nu$ and $\lambda$, so that $\nu$ determines the number of bound states while $\lambda$ determines the shape of the potential barrier and generates the resonances. In this paper we have concentrated on resonances with $L=0$. We have considered two cases: The Pöschl-Teller (PT) case, corresponding to $\lambda=1$, where no resonances appear, and the case of $\lambda=10$, to which we refer as the Ginocchio (G) case which displays resonances. The values of the $\nu$ parameter and the scale length have been chosen so that both potentials have only one bound state, with the same mean square radius and binding energy.

We have applied the THO method to discretize the con- tinuum in both cases. The Hamiltonian have been diagonalized in the THO basis, for a given number of states, and the eigenstates and eigenvalues have been obtained. Although there are small differences between the spectra of the PT and $\mathrm{G}$ cases, there is not a clear signal which allows us to identify the resonance in the $\mathrm{G}$ case. The same can be said about the wave functions of the eigenstates.

We have developed a procedure to obtain the state in the THO basis which represents the resonance. We start considering the eigenstates of the operator $\Delta^{2}=P h Q h P$, which is related to the matrix elements of the Hamiltonian which couple states within the THO basis with states outside it. As the size of the basis increases, we show that the expectation value of the Hamiltonian for the eigenstates of $\Delta^{2}$ decreases in general. However, when there are resonances, some eigenstates display an unexpected increase in the expectation value of the energy. These states, which are eigenstates of $\Delta^{2}$ and show a large expectation value of the energy, are identified with the resonances in the THO basis.

The THO resonance has a radial behavior within the potential well which is very similar to that of the continuum states with energies close to the resonance energy. Outside the potential, the THO resonance decreases smoothly. The THO resonance can be expanded in terms of states of the continuum, and it displays a structure which is consistent in position and width with a Breit-Wigner distribution.

We have investigated the completeness properties of the THO basis, both in the resonant and in the nonresonant case. When the number of states is infinite, the THO basis describes the complete continuum. Nevertheless, for a finite number of THO states, the description of states with low momentum is more accurate than that of high momentum. In the resonant case, however, the description of the continuum is more accurate in the range of the resonances than outside it. Regarding completeness in configuration representation, it is seen that, in the nonresonant case, smaller distances are better described than larger distances, while for the resonant case, the distances close to the potential barrier are best described.

We have investigated the coupling of the bound state to the continuum. It is found that, for the nonresonant case, the strength function is a smooth function of the momentum of the continuum state, while in the resonant case there is a characteristic resonant structure for the momenta close to the resonance. The THO basis, with a small number of states, describes accurately the strength function in both cases. Moreover, the THO resonance is found to describe the strength contribution at the energies of the resonance.

In summary, we have shown that the THO basis provides an adequate representation of the continuum, both resonant and nonresonant. It allows us to identify a single state that represent each resonance. The difference between the strength functions in resonant and nonresonant cases can be understood and calculated accurately in the THO basis.

We consider that the results presented here for a particular potential shape and just one bound state with $L=0$ should be applicable in more realistic cases. The extension to arbitrary potential shapes with ground state known either analytically or numerically, several bound states, and angular momentum different from zero is straightforward. The extension to cases 
in which the resonances have a many-body nature is more involved but it can be done. If the explicit expression of the Hamiltonian is known, then the matrix elements of $h^{2}$ can be evaluated, and the procedure outlined here can be directly applied. If one does not know the explicit form of the Hamiltonian but knows how to calculate matrix elements of the Hamiltonian in a many-particle configuration space, as it is the case, for instance, in the nuclear shell model, the method presented here can be used. One has to define a large basis (e.g., many harmonic oscillator shells) out of which a smaller set of states is selected (e.g., one harmonic oscillator shell) as an approximated basis for a reduced configuration space. Then, one can study the resonances in this reduced space with the method presented here. Let us call schematically $|\ell\rangle$ to the states in the large basis and $|s\rangle$ to the basis states in the small basis. The matrix elements of $\Delta^{2}$ are obtained as

$$
\begin{aligned}
\left\langle s^{\prime}\left|\Delta^{2}\right| s\right\rangle & =\left\langle s^{\prime}\left|h^{2}\right| s\right\rangle-\sum_{s^{\prime \prime}}\left\langle s^{\prime}|h| s^{\prime \prime}\right\rangle\left\langle s^{\prime \prime}|h| s\right\rangle \\
& =\sum_{\ell}\left\langle s^{\prime}|h| \ell\right\rangle\langle\ell|h| s\rangle-\sum_{s^{\prime \prime}}\left\langle s^{\prime}|h| s^{\prime \prime}\right\rangle\left\langle s^{\prime \prime}|h| s\right\rangle .
\end{aligned}
$$

Once the $\Delta^{2}$ matrix is constructed from the matrix elements of $h$ one can proceed as shown in this paper to study the resonances in the restricted space.

\section{ACKNOWLEDGMENTS}

This work was supported in part by the Spanish MCyT under Project Nos. FPA2002-04181-C04-04 and BFM200203315. We acknowledge useful discussions with R. Liotta and C. H. Dasso.
[1] G. Gamow, Z. Phys. 51, 204 (1928); 52, 510 (1928).

[2] N. Austern, Y. Iseri, M. Kamimura, M. Kawai, G. Rawitsher, and M. Yahiro, Phys. Rep. 154, 125 (1987).

[3] M. Rotenberg, Adv. At. Mol. Phys. 6, 233 (1970).

[4] F. Antonsen, Phys. Rev. A 60, 812 (1999).

[5] R. Szmytkowski and B. Zywicka-Mozejko, Phys. Rev. A 62, 022104 (2000).

[6] E. Hiyama, Y. Kino, and K. Kamimura, Prog. Part. Nucl. Phys. 51, 223 (2003).

[7] M. Moshinsky and Y. Smirnov, The Harmonic Oscillator in Modern Physics (Harwood Academic Publishers GmbH, Amsterdam, 1996).

[8] F. Perez-Bernal, I. Martel, J. M. Arias, and J. GomezCamacho, Phys. Rev. A 63, 052111 (2001).

[9] I. Martel, F. Perez-Bernal, M. Rodríguez-Gallardo, J. M. Arias, and J. Gomez-Camacho, Phys. Rev. A 65, 052708 (2002).
[10] F. Perez-Bernal, I. Martel, J. M. Arias, and J. GomezCamacho, Few-Body Syst., Suppl. 13, 217 (2001).

[11] A. M. Moro, J. M. Arias, J. Gomez-Camacho, I. Martel, F. Perez-Bernal, R. Crespo, and F. Nunes, Phys. Rev. C 65, 011602 (2002).

[12] M. Stoitsov, P. Ring, D. Vretenar, and G. A. Lalazissis, Phys. Rev. C 58, 2086 (1998).

[13] M. V. Stoitsov, W. Nazarewicz, and S. Pittel, Phys. Rev. C 58, 2092 (1998).

[14] M. V. Stoitsov, J. Dobaczewski, P. Ring, and S. Pittel, Phys. Rev. C 61, 034311 (2000).

[15] J. N. Ginocchio, Ann. Phys. (N.Y.) 152, 203 (1984); 159, 467 (1985).

[16] G. Pöschl and E. Teller, Z. Phys. 83, 143 (1933).

[17] A. U. Hazi and H. S. Taylor, Phys. Rev. A 1, 1109 (1970). 